Table II. Cost data for VLT and ST compared with the ESO $3.6 \mathrm{~m}$.

Investment, M SwFr

Capital charge, M SwFr/a

Operation, M SwFr/a

Annual Cost, M SwFr

Observing time, s/a

Rel. observing cost, SwFr/s

Rel. cost/photon

$\begin{array}{ccc}\text { ESO, 3.6 m } & \text { VLT, } 16 \mathbf{~ m} & \text { ST, } 2.4 \mathbf{~ m} \\ 70 & 200 & 1000 \\ 5 & 13 & 70 \\ 20 & 30 & 80 \\ 25 & 43 & 150 \\ 4 \times 10^{6} & 4 \times 10^{6} & 9 \times 10^{7} \\ 6 & 11 & 17 \\ 10 & 1 & 25\end{array}$

spectroscopic studies designed to give reasonable spectral resolution for objects in the magnitude range $18-23$, as well as in the study of objects with rapid time variations, a bigger collecting area is essential. Moreover, if extended objects like galaxies with angular sizes in excess of 1 arc $s$ are under investigation, much of the advantage of the ST is lost (unless angular resolution is needed). These then are the essential arguments for the projects now being studied: in Europe for a terrestrial $16 \mathrm{~m}$ telescope - the Very Large Telescope (or VLT) and in the US for a $25 \mathrm{~m}$ telescope - the Next Generation Telescope (or NGT).

Observations that would be made with the VLT include: 1) Redshifts of faint distant galaxies; comparatively small differences in apparent magnitude for the same redshift correspond to very different models of the Universe. 2) Sharp absorption lines in the spectra of distant quasars which may be due to matter in intergalactic space between the quasar and us; of value in evaluating models of the Universe and also galaxy formation. 3) Polarization structure of the light emitted from pulsars which is essential for ascertaining the conditions in the very strong magnetic fields around the neutron star; various $X$-ray sources show millisecond time scale structure in their light emission. 4) Composition of stars and regions of ionized gas in nearby galaxies; current information in this area is limited to our own galaxy as well as of globular clusters in other galaxies; the distribution of these objects; spectropolarimetric studies of quasars and stars...

Construction of a $16 \mathrm{~m}$ telescope poses quite new problems, as it is clear the philosophy of building a single massive light collector that has applied up to now must be abandoned. Two approaches are open: an array of smaller telescopes, e.g. four $8 \mathrm{~m}$ or sixteen $4 \mathrm{~m}$ instruments; a single instrument with a light composite mirror, the profile of which is continuously and dynamically controlled. With the multi-instrument approach, each telescope can be equip- ped with its own detection equipment. The array is then flexible in operation but expensive and limited to incoherent measurements. Alternatively it can be equipped with an optical system for light combination that allows coherent measurements but reduces materially the efficiency of photon collection because of mirror losses. Some compromise between the two is also possible. ESO, with its background of a computerized pointing and data collection system on the $3.6 \mathrm{~m}$ telescope at La Silla, is concentrating for the present on the single dish approach which it believes could be realized by 1990 .

Cost of such a large instrument is for the moment necessarily speculative, but realistic extrapolations from current experience suggest that an array could be built for about $250 \mathrm{M}$ SwFr and that this is not the most economic approach. Capital cost is not, however, the sole criterion upon which a judgment should be based, and ESO has computed the relative costs of the present $3.6 \mathrm{~m}$ as against the VLT and ST in terms also of cost per unit observing time and cost per photon detected. Calculations are based on observations of a 20th magnitude star. From Table II it can be seen that although the ST is expensive in capital investment, it has a on detachment to IRAM from CERN, Geneva.
Millimetre-wave astronomy is a relatively new branch of astronomy as the first good quality millimetre receiver was set in operation only in 1969. Since that time, the field has yielded an impressive blossom of results in spite of the still modest size of the instruments: most of the 50 interstellar molecules known today have been discovered through observations of several hundred lines in this wavelength range, and also many isotopic substitutions. These molecules, which much greater utilization factor. In the last line the efficiency of the VLT in cost per photon is evident.

\section{European VLBI Network}

Plans are now advanced for having a European Very Long Baseline Interferometer network. Essentially international, the project is ideally suited to a European cooperation where each country has its own observing facilities but the results come from a combination of all.

At present, data from existing stations are recorded separately on tapes with a caesium clock providing the time standard, but the process of combination is slow and tedious. Proposals have therefore ben made to ESA for a dedicated communications satellite which would allow the European VLBI to operate in real time. Moreover, it would lead to great progress in astrometry in particular in connexion with the problem of establishing a fundamental reference frame, and in geophysics by making possible the detection of crustal motions of the order of $1 \mathrm{~cm}$ over $1000 \mathrm{~km}$.

\section{Other Collaborative Projects}

In the European space programme there is under construction a large satellite for X-ray studies - Exosal and an infrared satellite - IRAS due for completion in 1980/81 that will, for the first time, make a full inventory of the infrared sky down to faint flux levels. Plans for other possible satellites include an astrometric satellite and an XUV satellite which would search for sources in the hard ultraviolet and soft X-ray range - a region where at present very little is known.

\title{
Radio Astronomy Projects in the Millimetre Range
}

A summary of his talk on the Franco-German project IRAM, prepared by Kjell Johnsen,

make up at least half the mass of the interstellar gas in the Galaxy, are mainly found in previously unknown massive and relatively dense molecular clouds which appear to be the principal sites of star formation. Radio line and continuum observations and radio observations of interstellar masers, as well as observations in the infrared and submillimetre range, have allowed preliminary mapping of some of these clouds and have revealed dense and hot spots which 
might be protostars. The discovery of molecules in external galaxies has also raised considerable interest.

Nuclei of galaxies and quasars are other astronomical objects whose continuum millimetre emission is sometimes very strong and rapidly variable, and there is a hope that millimetre astronomy will help us to understand their nature.

For such reasons, the French and German radio astronomers have for many years worked on plans for extending their observation facilities into the millimetre range. The French have focused their interest mainly on interferometer types of telescope, the Germans on single-dish telescopes. Difficulties were encountered in raising funds nationally, which led them to explore the possibility of cooperation between the two countries to achieve the same aim. This would have several advantages. The two national radio astronomy communities would each have access to two complementary instruments and it can be hoped that the total cost might be reduced by pooling forces. Last, but not least, this would be another scientific venture that fostered international cooperation.

Arguments of this kind were sufficiently convincing for the Centre National de la Recherche Scientifique (CNRS) and the Max-Planck-Gesellschaft zur Förderung der Wissenschaften (MPG) to start in 1973 to work out administrative and legal details for such a cooperation and the astronomers were encouraged to work on the details of the two instruments.

The original idea was to have the two telescopes on a common site, preferably on the continent of Europe. A siting far south is desirable to facilitate the observation of the centre of our Galaxy and as water vapour attenuates strongly $\mathrm{mm}$ waves, it is important to have a high altitude site, in a region with a good climate.

The promoters of the single-dish telescope, rather quickly homed in on a mountain peak, at a height of about
$3000 \mathrm{~m}$, in the Sierra Nevada in the South of Spain, close to the City of Granada. However, this region has one severe drawback: it does not have room for an interferometer, which requires a plateau, flat over about $1 \mathrm{~km}$.

The best site found in Western Europe for the interferometer was Plateau de Bure, at an altitude of about 2500 m, somewhat South of Grenoble.

This very natural development was at the time a disappointment for the cooperation. However, in radio astronomy it is not uncommon to have a joint exploitation of geographically dispersed telescopes. A typical example is the National Radio Astronomy Observatory (NRAO) in the USA, and a similar solution was recommended for the joint French/German institute by a working party of "three wise men", i.e. to place the single-dish telescope in the Sierra Nevada, the interferometer at Plateau de Bure and to co-ordinate the operation and the scientific programme from a Central Institute, preferably near one of the telescopes; Grenoble was recommended.

Plans along these lines were finally adopted by CNRS and MPG at the end of 1978, under the name of Institut de Radioastronomie Millimétrique (IRAM).

\section{$30 \mathrm{~m}$ Single-Dish Telescope}

The main parameters of the singledish telescope are summed up in Table I. For reasons of cost, it has been decided not to cover the antenna by an astrodome or radome. This means a stronger antenna structure and deicing must also be provided. This adds to the cost of the antenna, but is still cheaper than a solution with an astrodome or radome.

The antenna will be designed and constructed under the responsibility of the Max-Planck-Institut für Radioastronomie (MPIfR) in Bonn on a contract with IRAM. Most of the receiver and back end work will be the responsibility of IRAM, in Grenoble.

\section{Interferometer}

The main parameters of the interferometer are summed up in Table II. Again no astrodome or radome is foreseen. This observatory will be designed and constructed under the responsibility of the Project Office of IRAM in Grenoble.

\section{Central Institute}

A Central Institute for IRAM will be established in Grenoble. Its general long-term purpose will be to ensure an efficient operation of the observing facilities and scientific and technical co-ordination of the research programme.

Its immediate task will be to build up a project office and technical groups that can take on the design and construction of various elements of the telescopes. IRAM will be permitted to grow up to 80 staff positions. During the construction period however, it is hoped to be able to draw on additional manpower assistance from the astronomy communities in France and Germany.

It is not expected, especially in the operation phase, that all staff will be situated at the Central Institute. Some will be stationed in Granada, and some will be needed at or near Plateau de Bure. IRAM will have its own building in Grenoble in the same area as the Institut Max von Laue - Paul Langevin (ILL) and other research institutes.

\section{Cost and Time Schedule}

The total capital during the construction phase of IRAM is estimated to come to approximately $160 \mathrm{MFF}$, about equally divided between the single-dish telescope and the interferometer. CNRS and MPG share these expenses equally. Much of the MPG contribution comes from the Volkswagen Foundation.

It is planned to put the single-dish telescope into operation in 1982,

\begin{tabular}{|c|c|}
\hline TABLE I & \\
\hline Reflector Diameter & $30 \mathrm{~m}$ \\
\hline Antenna Mounting & Fully steerable \\
\hline r.m.s. surface accuracy & $0.07 \mathrm{~mm}$ \\
\hline Receiver systems & $\begin{array}{rc}22 & \mathrm{GHz} \\
43 & \mathrm{GHz} \\
80-120 \mathrm{GHz} \\
140-170 \mathrm{GHz} \\
(230 \mathrm{GHz})\end{array}$ \\
\hline Beam width (at $115 \mathrm{GHz}$ ) & 20 arc s \\
\hline $\begin{array}{l}\text { Max. wind speed for } \\
\text { observation }\end{array}$ & $14 \mathrm{~m} / \mathrm{s}$ \\
\hline
\end{tabular}

\section{TABLE II}

Number of antennas

Diameter of antennas

r.m.s. surface accuracy

Antenna mounting

Maximum baseline

Receiver systems
3

$12-15 \mathrm{~mm}$

$0.07 \mathrm{~mm}$

Fully steerable and movable

$1.5 \mathrm{~km} \times 0.8 \mathrm{~km}$

$22 \mathrm{GHz}$

$43 \mathrm{GHz}$

$80-120 \mathrm{GHz}$

$140-170 \mathrm{GHz}$

(230 GHz)

Beam width (at $115 \mathrm{GHz}$ )

2 arc s (with $400 \mathrm{~m}$ baseline) 
while the completion of the interferometer is foreseen for 1986 only. This is determined by the possible inflow of funds and not by technical considerations. However, since the interferometer consists of three antennas, it is foreseen to proceed in stages, such that the first antenna at Plateau de Bure can be made operational by 1983 .

\section{Present Status}

The negotiations with Spain on the Sierra Nevada site are in the final stage. At first it was hoped to place the antenna very close to the Pico de Veleta (at $3300 \mathrm{~m}$ ), but this met environmental objections. A satisfactory site near-by (at $2900 \mathrm{~m}$ ) has been offered instead. In exchange for offering a site and the local headquarters in Granada, Spanish astronomers will get about $10 \%$ observation time at both observatories, and will become an integrated part of the radio astronomy community utilizing the instruments.
The MPIfR are almost ready to place the order for the construction of the large dish and the design of the interferometer antennas is in the initial stage. Here the first task is to determine the final diameter to fit the allocated funds.

The project office will be established in Grenoble in May this year, in a temporary building put at IRAM's disposal by the University of Grenoble. By the end of 1979 , it is hoped that IRAM will have 20-25 people in Grenoble, with several technical groups active, in particular, the receiver group.

\section{Concluding Remarks}

Another international research institute is in the process of being born. It has for the time being only two funding partners, which constitute both advantages and disadvantages. It is to be hoped that more partners will join later to make IRAM a truly European Institute.

\section{TeV Fixed Target Proton Synchrotron Projects}

A shortened version of the paper prepared by K. P. Myznikov of Moscow in the name of a team of over $\mathbf{5 0}$ physicists from institutes in the USSR. At the seminar, the paper was introduced by $\mathrm{V}$. Sidorov.

The Institute for High Energy Physics' Accelerating and Storage Complex (UNK), being developed for still deeper studies in high energy physics is a $3 \mathrm{TeV}$ superconducting proton synchrotron with the prospect of obtaining colliding beams in the future. The design study has already been

Lay-out of UNK in relation to the present Serpukhov machine.

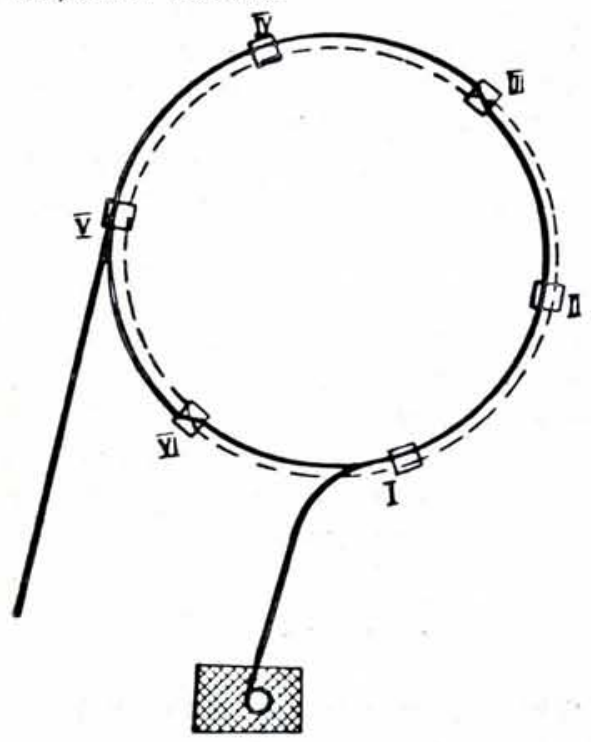

completed, and the basic parameters are listed in Table I.

UNK is a two-stage accelerator, wherein the first stage is a proton synchrotron with conventional magnets, designed to stack and pre-accelerate protons up to $400 \mathrm{GeV}$, and the second an accelerator utilizing superconducting magnets for achieving the highest energies. Both accelerators are located in a common tunnel. The existing $70 \mathrm{GeV}$ accelerator at Serpukhov (U-70), the intensity of which is planned to be increased up to $5 \times 10^{13} \mathrm{ppp}$, is to be used as an injector into UNK.

The two-stage system simplifies the superconducting accelerator operating mode substantially. Beam preacceleration in the first stage makes it possible to reduce the effects of residual fields, and the decrease in beam dimensions reduces the severity of the field specifications imposed on the superconducting magnets. This is also helped by having single-turn beam injection into the central orbit of the second stage. Beam stacking takes place in the first stage, so freeing the superconducting accelerator from inevitable particle losses.
Other advantages are to be found in the two-stage system. It allows the maximum use to be made of the injector intensity and the availability of two rings leaves open the option of a $0.4 \mathrm{TeV}$ and $3 \mathrm{TeV}$ proton-proton colliding beam facility yielding 2.2 $\mathrm{TeV}$ energy in the centre of mass. The ring of the first stage can also be used to stack pure beams of electrons, antiprotons, etc.

Fig. 1 shows the layout of UNK, with the main ring centre, taking into account geological restraints, located $6.5 \mathrm{~km}$ from the U-70 accelerator centre. Dimensions of the tunnel are chosen with in mind the possibility of installing there a superconducting storage ring. This will enable a $3 \mathrm{TeV}$ proton beam to be stacked and made to collide with a beam of the same energy accelerated in the pulse superconducting ring. As a result, the ppcollision energy can be increased up to $6 \mathrm{TeV}$ in the c.m.s.

The UNK project is designed with its future development in view. Six straight sections, each $485 \mathrm{~m}$ long, are introduced into the structure of both stages (Fig. 1). A beam from $\mathrm{U}-70$ is injected into section I, where the beam transfer system into the second stage is also located. The accelerated particles are extracted from section V. The accelerating systems are placed in section II, and the beam abort systems in section IV. Sections III and IV are reserved for colliding beams. The layout of the accelerator and storage rings in the tunnel and the construction of the straight sections are chosen so that the intersections of the maximum energy beams would be obtained more easily. Fig. 2 presents a schematic view of the UNK tunnel cross-section and the position of the magnets there.

Great importance has been attached to developing for the superconducting magnet units protection measures against radiation. Stacking at $70 \mathrm{GeV}$ and beam pre-acceleration take place in the first stage

Cross-section of the tunnel showing: 1 , first stage; 2 , second; 3 , storage ring.

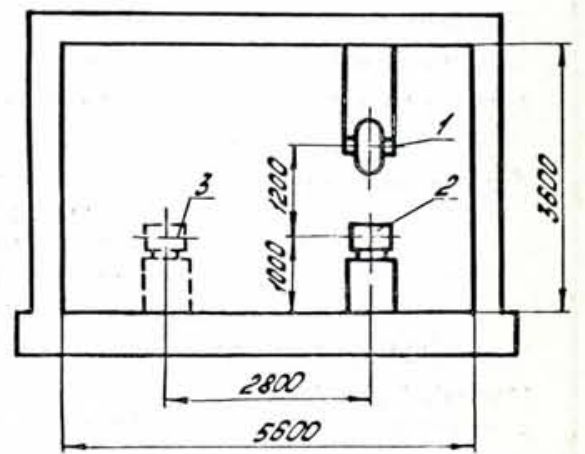

